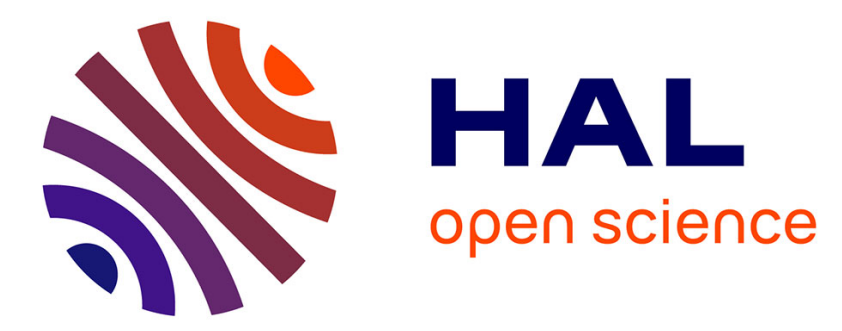

\title{
Large mode area aperiodic fiber designs for robust singlemode emission under high thermal load
}

Romain Dauliat, Enrico Coscelli, Federica Poli, Dia Darwich, Aurélien Benoit, Raphaël Jamier, Kay Schuster, Stephan Grimm, Annamaria Cucinotta, Stefano Selleri, et al.

\section{To cite this version:}

Romain Dauliat, Enrico Coscelli, Federica Poli, Dia Darwich, Aurélien Benoit, et al.. Large mode area aperiodic fiber designs for robust singlemode emission under high thermal load. SPIE Photonics Europe, Apr 2015, Prague, Czech Republic. pp.950709. hal-01250202

\section{HAL Id: hal-01250202 \\ https://hal.science/hal-01250202}

Submitted on 4 Jan 2016

HAL is a multi-disciplinary open access archive for the deposit and dissemination of scientific research documents, whether they are published or not. The documents may come from teaching and research institutions in France or abroad, or from public or private research centers.
L'archive ouverte pluridisciplinaire HAL, est destinée au dépôt et à la diffusion de documents scientifiques de niveau recherche, publiés ou non, émanant des établissements d'enseignement et de recherche français ou étrangers, des laboratoires publics ou privés. 


\title{
Large mode area aperiodic fiber designs for robust singlemode emission under high thermal load
}

\author{
Romain Dauliat ${ }^{a, b}$, Enrico Coscelli ${ }^{c}$, Federica Poli $^{c}$, Dia Darwich $^{a}$, Aurélien Benoît ${ }^{a, d}$, Raphaël \\ Jamier $^{a}$, Kay Schuster ${ }^{b}$, Stephan Grimm ${ }^{b}$, Annamaria Cucinotta ${ }^{c}$, Stefano Selleri ${ }^{c}$, François \\ Salin $^{d}$ and Philippe Roy ${ }^{a}$ \\ ${ }^{a}$ Xlim research institute, UMR CNRS/Université de Limoges n7252, 123 avenue Albert \\ Thomas, 87060 Limoges Cedex, France; \\ ${ }^{b}$ Institute for Photonic Technology, Albert Einstein Straße 9, 07745 Jena, Germany; \\ ${ }^{c}$ Information Engineering Department, University of Parma, I-43124 Parma, Italy; \\ ${ }^{d}$ Eolite systems, 11 avenue de la Canteranne, 33600 Pessac, France;
}

\begin{abstract}
In this paper, we investigate the potential of various large mode area fibers under thermal load, that is the state-of-the-art air-silica large pitch fibers, as well as the recently devised symmetry-reduced photonic crystal fiber and aperiodic all-solid by carefully considering the degrees of freedom offered all along the fiber fabrication. This work aims to discuss the mode filtering ability of these structures in regard to the power scaling and to confirm their potential for robust singlemode operation at high power level. Structural principles contributing to improve their performances such as the impact of air holes / solid inclusions size will be presented. We also intend to establish that the range of average absorbed/output power for which a robust singlemode operation is available can be shifted to fulfil user requests in term of power range.
\end{abstract}

Keywords: Microstructured optical fibers, aperiodic Large-Mode-Area fibers, fiber lasers and amplifiers, singlemode operation, thermal issues

\section{INTRODUCTION}

For two decades now, fiber lasers have been subject to a perpetually revived effervescence for power scaling driven by their versatility, making them appropriate for a wide range of applications. As a consequence, a tremendous ascent in emitted power has been performed notably thanks to Yb-doped fibers. This led to attain up to $10 \mathrm{~kW}$ in $\mathrm{CW}$ regime ${ }^{1}$ or $\mathrm{GW}$ peak power in femtosecond pulses regime. ${ }^{2}$ However, further progress is often synonymous of evidence of new limitations. This rule applies also to optical fibers, especially when operating in high power regime. Although the effects of many disruptive phenomena such as non-linear impairments or dielectric breakdown have already been mitigated thanks to the use of Very-Large-Mode-Area (VLMA) fibers, other significant detrimental issues must still be solved.

Indeed, fiber designers and experimenters must nowadays deal with two major obstacles intrinsic to highpower fibers laser/amplifiers. First of all, the so-called photodarkening, which manifests itself as a temporal growth of background losses, is responsible of a reduction of the laser efficiency caused by the generation of color centers. ${ }^{3}$ This mechanism is especially detrimental under strong population inversions, ${ }^{4}$ being thus deleterious to amplification schemes. On the other hand, high power operation implies a strong heat load of the gain medium by non-radiative relaxation, resulting in thermal-induced problems even with low quantum defect ions. Among these thermal effects, modal instabilities represent the main key hurdle for a further power scaling. ${ }^{5}$ This phenomenon strongly degrades the spatial mode quality when the output average power exceeds a certain threshold at which the Fundamental Mode (FM) and one High Order Mode (HOM) transfer back and forth their energy in a ms timescale. While photodarkening can be efficiently mitigated by adjusting the material composition of the active core ${ }^{6}$ modal instabilities can be controlled by balancing material properties, that is absorption and laser efficiency, with the ability of the fiber structure to discriminate High-Order-Modes (HOMs).

Further author information: (Send correspondence to)

E-mail: romain.dauliat@ipht-jena.de / romain.dauliat@xlim.fr, Telephone: +49(0)3641 206207 / +33 (0)5 55457268 
In this context, several strategies have been adopted to improve the HOM rejection in VLMA fibers, thus making more robust their singlemode behaviour. Among the most relevant approaches, many geometries belonging to the class of leaky structures can be identified. ${ }^{7-10}$ These designs take benefit of the inherent stronger leakage of HOMs in comparison to the fundamental mode to perform an efficient spatial mode filtering while still ensuring a strong interaction of the FM with the gain region. Hence, some flexibility is offered in designing tailored microstructured cladding thanks to the stack and draw technique. Thus, it has been emphasized that the widespread hexagonal photonic crystal cladding (PCC) structuration is not strictly required. For instance, a pentagonally arranged cladding ${ }^{11}$ as well as an aperiodic cladding array provide higher levels of HOMs delocalization than the standard PCC. ${ }^{12}$ The absence of cladding symmetry helps in avoiding the resonance of HOMs on the cladding and part of the mode coupling by minimizing the spatial overlap between the core fundamental mode and the cladding ones. Thereby, in order to alleviate the harmful impact of thermal-induced processes on the beam quality at high power levels, our goal in this publication is to deeply analyse the performances of these leaky fibers with the power scaling, i.e. under the influence of an increasing thermal load value and to demonstrate the role of non-periodic structuration.

\section{DESCRIPTION OF THE FIBER DESIGN}

Leaky fibers designs are mostly based on the stack and draw technique. Starting from a set of circular canes stacked in accordance with the final fiber design, the drawing enables to preserve the fiber geometry except that the interstitial air holes are collapsed, yielding commonly in an array of hexagonal elements. Although never commented up to now, the stack of Large Pitch Fiber (LPF) has undergone a significant change over the last 4 years. In fact, initially they had a unique cell constituting the active core. ${ }^{13}$ Since then, they passed by 7 cells core $^{14}$ to finally end up with the use of 19 actively doped elements structuring the core. ${ }^{15}$ This yields in fiber stacks made respectively of 19,61 or 169 canes as represented in Fig.1. An air-clad, in charge of guiding the pump radiation, and a thick jacket, ensuring the rigidity of the fiber, are generally added around the structure. The small gap appearing in between the stack and the air clad corresponds to the tube used during the stack drawing into cane. The influence of the stack geometry will be discussed throughout this paper.

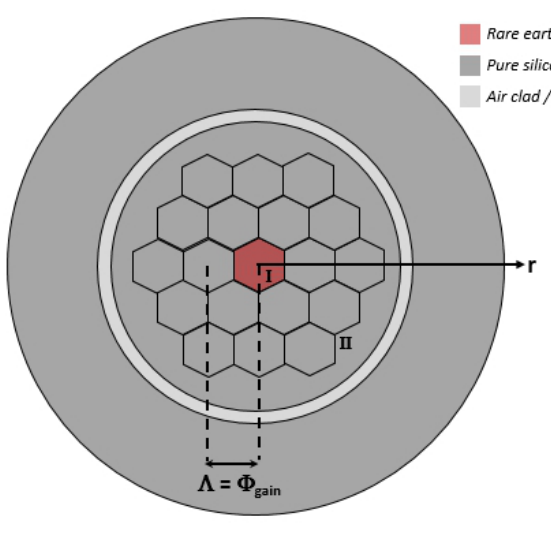

(a)

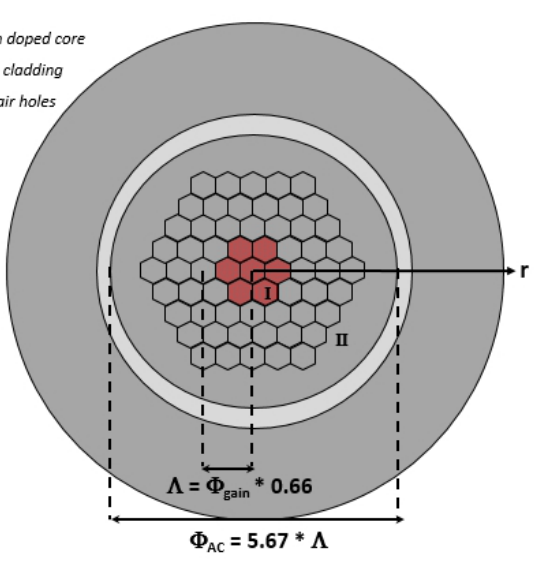

(b)

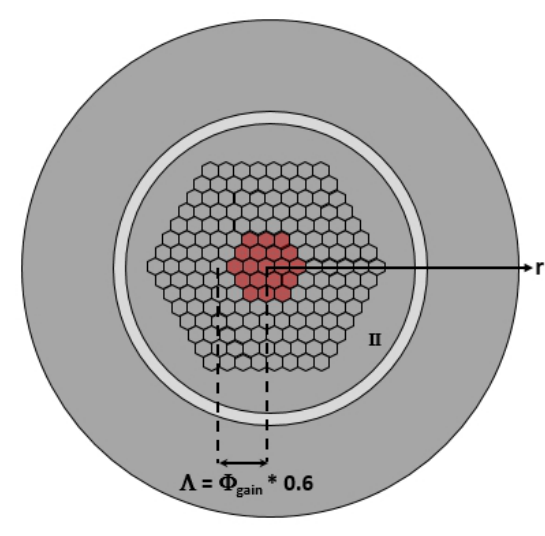

(c)

Figure 1. Graphical representations of fiber microstructured arrays obtained by resorting to the "Stack \& draw" technique. Three kinds of arrangement based on (a) 19 canes ,(b) 61 canes or (c) 169 canes are commonly made. This design are used respectively with (a) 1, (b) 7 and (c) 19 actively doped rods - in red - resulting in different gain area at equivalent air clad diameter. The dark grey region represents the pure silica and the clear grey correspond to the air.

All the geometry have been defined so as to be adaptive with the pitch $\Lambda$, being the distance from the center of the gain region to the one of the first passive element in the gain region surrounding as shown in Fig.1. For each case, the relation between the pitch $\Lambda$ and the diameter of gain is reported in Fig.1. Moreover, the air-clad diameter is set to $5.67^{*} \Lambda$ to coincide with state-of-the-art air-silica LPF. It is important to underline that for 
equivalent $\Lambda$ or air-clad diameters, the extension of the gain region will be increasing with the number of active cells. To illustrate this fact, one may look to the value of the ratio between the gain and air clad areas, which are $0.03434,0.06$ and 0.07249 for respectively 1,7 and 19 active cells. This means that for an equivalent linear gain, the rare-earth ions concentration could be half less in the 19 cells case than for a unique active element. Hence, our analysis will demonstrate how the stack geometry can have a major impact on the performance of VLMA fibers in high power regime.

\section{MODELLING OF MODAL CONTENT IN ACTIVE FIBERS UNDER HEAT LOAD}

The heat generated all along the fiber length is responsible for Refractive Index Changes (RIC) and modal instabilities which can cause a quality degradation of the emitted beam. Indeed, owing to the non-radiative relaxations, part of the absorbed pump power is released as heat, yielding a thermal loading of the fiber ultimately impacting his refractive index profile through a thermo-optic mechanism. For this reason, it is essential to consider this thermal contribution in computing the modal content of optical fibers, especially into VLMA fibers which are highly sensitive to index variations. ${ }^{13}$

As into our previous work, ${ }^{9}$ a commercial full-vector finite-element solver was employed to determine the fiber mode content whereas a home-made program was coupled to it for considering the steady state heat equation. The temperature distribution $\mathrm{T}(\mathrm{r})$ across the fiber section is thus described by the following equation:

$$
\begin{array}{cc}
\frac{1}{r} \frac{\delta}{\delta r}\left(r \frac{\delta T(r)}{\delta r}\right)=-\frac{Q_{0}}{k_{S i}} & \text { for } r \leq R_{\text {gain }} \\
\frac{1}{r} \frac{\delta}{\delta r}\left(r \frac{\delta T(r)}{\delta r}\right)=0 & \text { elsewhere }
\end{array}
$$

where $\mathrm{Q}_{0}=\mathrm{q} / \mathrm{A}_{\text {gain }}$ stands for the generated heat density in $\mathrm{W} / \mathrm{m}^{3}$, and represents the heat load ' $\mathrm{q}$ ' in $\mathrm{W} / \mathrm{m}$ deposited across the area of the gain region $\mathrm{A}_{\text {gain }}$. The thermal conductivity $\mathrm{k}$ is fixed at $\mathrm{k}_{S i}=1.38 \mathrm{~W} /(\mathrm{m} . \mathrm{K})$ for the pure silica. In the air-clad, the thermal conductivity is fixed to $0.086 \mathrm{~W} /(\mathrm{m} . \mathrm{K}) .{ }^{9}$ Then, the heat dissipation is assumed to occur by natural convection at the fiber outer surface $R_{\text {out }}$, the diameter of which is set at $1 \mathrm{~mm}$. This behaviour is numerically described by using the Newton's law of cooling:

$$
\frac{\delta T\left(r=R_{\text {out }}\right)}{\delta r}=\frac{h}{k_{S i}} *\left[T_{\text {cool }}-T\left(R_{\text {out }}\right)\right]
$$

where the convection coefficient $\mathrm{h}$ is of $80 \mathrm{~W} /\left(\mathrm{m}^{2} . \mathrm{K}\right)$ and $\mathrm{T}_{\text {cool }}$ is the ambient temperature. By using jointly the two above-mentioned equations, the thermal-induced RIC can then be determined as $\Delta \mathrm{n}=\beta * \Delta \mathrm{T}$, where $\beta$, the thermo-optic coefficient of the silica, equals $1.16 \cdot 10^{-5} /{ }^{\circ} \mathrm{K}$.

Thence, the calculation of the modal content into fibers undergoing a consistent heat load can be performed. Two criteria were formalized in this work so as to quantify an efficient singlemode operation: ${ }^{16,17}$

- an efficient amplification is established only if the fundamental $\mathrm{LP}_{01}$ overlaps by more than $80 \%$ the gain area:

$$
\Gamma_{01} \geq 80 \%
$$

- thereafter, an effective singlemode operation is achieved if the most competitive HOM is by at least $30 \%$ less confined in the gain area than the $\mathrm{LP}_{01}$ :

$$
\Delta \Gamma=\Gamma_{01}-\Gamma_{H O M} \geq 30 \%
$$

Although this approach is empirical, this is of great use in studying analytically the performance of fiber design. Later, the range on which both criteria are satisfied will be referred as to 'efficient singlemode amplification' range. 


\section{THERMALLY GUIDING FIBERS}

As the thermal load impacts directly the refractive index profile of an active fiber, we decided first to observe how it is involved into the modes confinement of simple clad 'Thermally Guiding Fibers (TGF)'. These designs, represented in Fig.1, are composed of hexagonally shaped gain regions labelled 'I' - made of 1, 7 or 19 active cells as into VLMA fibers which are embedded into a pure silica material named 'II'. A strict index matching between the gain material and the pure silica is considered here as VLMA fibers are well-known to be little tolerant to any index mismatching. Both regions are thus set to an exact same refractive index of 1.45 at $1.032 \mu \mathrm{m}$. The $7-\mu \mathrm{m}$ thick air-clad acts thus as the unique cladding since no microstructuration is present for now.

\subsection{Evolution of the thermal-induced refractive index change}

To get a better understanding on how the modal content evolves with the heat load, it is essential to observe the behaviour of the thermal induced refractive index change. By reformulating equations 1 to 3 , the temperature profile across the core and the cladding can be expressed as:

$$
\begin{gathered}
T_{I}(r)=T_{0}-\frac{Q_{0} r^{2}}{4 k_{S i}} \quad \text { for } 0 \leq r \leq R_{\text {gain }}, \\
T_{I I}(r)=T_{0}-\frac{Q_{0} R_{\text {gain }}^{2}}{2 k_{S i}} \cdot\left[\mathbf{0 . 5}+\ln \left(\frac{\mathbf{r}}{\mathbf{R}_{\text {gain }}}\right)\right] \quad \text { for } R_{\text {core }} \leq r \leq R_{A C}
\end{gathered}
$$

stating a quadratic evolution of the temperature into the gain region and a logarithmic decay elsewhere. $\mathrm{T}_{0}$ is the temperature at the core center and $R_{A C}$ the inner radius of the air-clad. From these equations, the refractive index change across the gain region and the whole guiding structure can be expressed as follows:

$$
\begin{gathered}
\Delta n_{\text {core }}=\beta * \frac{q}{4 \pi k_{S i}} \\
\Delta n_{\text {guide }}=\beta * \frac{q}{2 \pi k_{S i}} *\left(0.5+\ln \frac{R_{A C}}{R_{\text {gain }}}\right)
\end{gathered}
$$

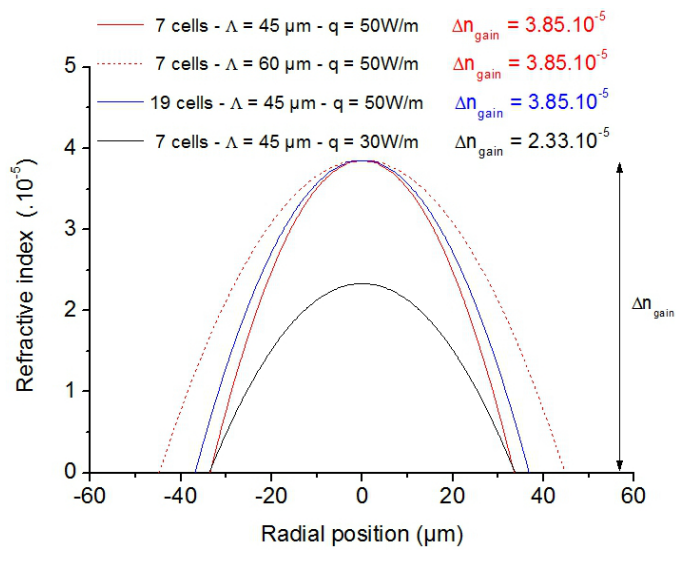

(a)

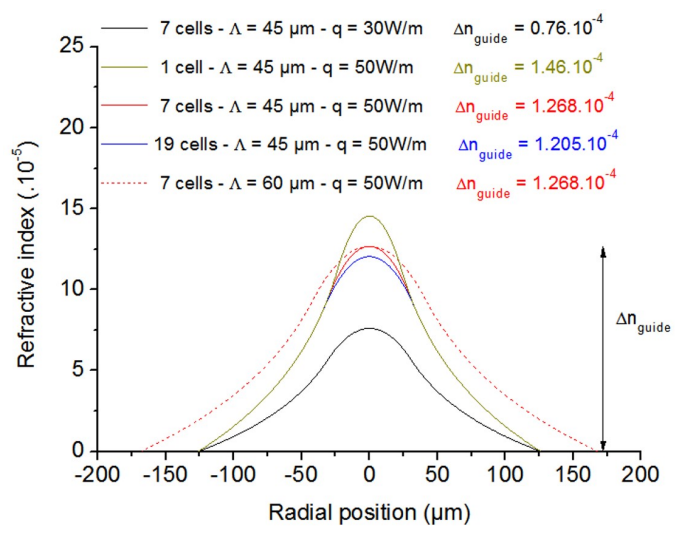

(b)

Figure 2. Refractive index profiles induced (a) across the gain region $\left(\Delta \mathrm{n}_{\text {gain }}\right)$ and (b) the whole guiding structure $\left(\Delta \mathrm{n}_{\text {guide }}\right)$ for different pitch $\Lambda$, heat load ' $\mathrm{q}$ ' or fiber structure. All the curves are normalized to the refractive index at the edge of (a) the gain region and (b) the air-clad. 
It is worth noting that the refractive index across the gain region is independent from the diameter of the doped area, as indicate in Eq.8 and shown in Fig.2(a). Indeed, in this region, the RIC is only related to the heat load 'q', and through it the absorbed pump power since $\mathrm{q}=\mathrm{P}_{a b s} *\left(1-\eta_{\text {quantum }}\right)$, with $\eta_{\text {quantum }}$ being the quantum efficiency of the gain medium. However, the dimension of the gain area plays a role in the heat diffusion process towards the fiber outer boundary. The ratio gain to clad area ratio $A_{\text {gain }} / A_{A C}$, which is different for each of the TGF, influences directly the RIC implies through the inner cladding. As an example, the RIC induced at different heat load, pitch $\Lambda$ and into different TGF designs are presented in Fig.2. The proportional increase of the RIC with the heat load is well displayed.

\subsection{Mode content in thermally guiding fibers}

When looking to the three TGF designs, the only noticeable disparity relies on their gain to clad ratio: $A_{\text {gain }} / A_{A C}$, whose value is $0.03434,0.06$ and 0.07249 for respectively a 1,7 and 19 active cells fiber. As previously demonstrated, this implies a quite moderate difference on the thermal induced RIC. Nevertheless, the performances of the thermally guiding fibers have been studied to establish a reference for this work.

Although the change in refractive index $\Delta n_{\text {core }}$ induced by the heat load is independent from the dimension of the gain region, an extension of the doped area will still contribute to confine much strongly each of the guided modes at a constant heat load 'q'. Thus, the study of thermally guiding fibers described in Fig.1 have been carried out in order to evidence the inherent potential of the heat load in confining modes, thus worsening the quality of the emitted beam. Fig.3 reports the evolution of the fundamental $\mathrm{LP}_{01}$ mode overlap factor in regard to the pitch $\Lambda$ and the heat load 'q'. The above mentioned criteria to identify the efficient singlemode amplification behaviour are displayed on these graphs by thick lines. In particular, a solid line is used for the limit of the efficient amplification $\left(\Gamma_{01}=80 \%\right)$ and a dashed one for an effective singlemode propagation $\left(\Delta \Gamma_{01}>80 \%\right)$.

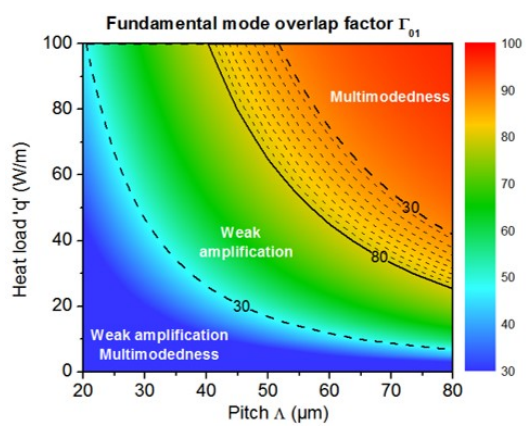

(a)

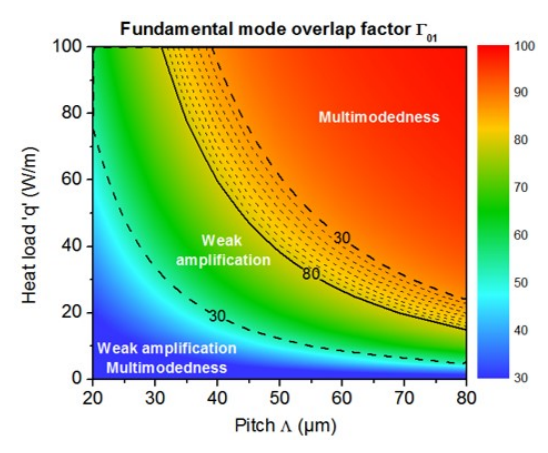

(b)

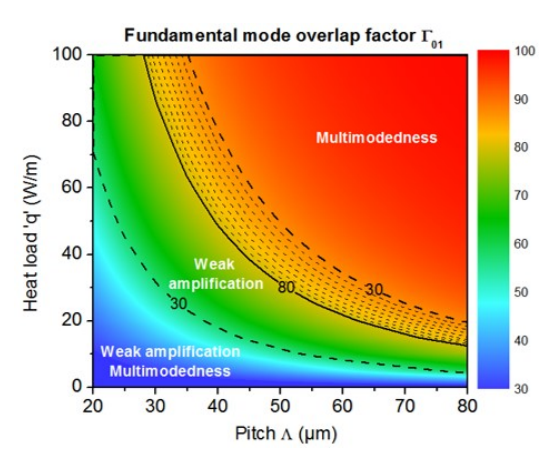

(c)

Figure 3. Evolution of the fundamental mode overlap factor with the heat load 'q' and the pitch $\Lambda$ into (a) a 1 cell TGF, (b) 7 cells TGF and (c) 19 cells TGF. The thick solid/dashed black lines represents respectively the limit of each abovementioned criteria: an efficient amplification $\Gamma_{01}=80 \%$ and an effective singlemodedness criteria: $\Delta \Gamma_{01}>80 \%$. The dashed area represents the region of effective singlemode amplification.

First of all, notice that even without any microstructuration of the inner cladding, an effective singlemode amplification can be achieved over a range of heat load values of about $30 \mathrm{~W} / \mathrm{m}$, equivalent to $120 \mathrm{~W}$ of emitted power in an Yb-doped fiber amplifier independently of the pitch. However, this performance can only be achieved under significant heat load or with large diameter of gain area. Indeed, since no microstructuration is employed to confine the radiation into the gain region, the overlap of the $\mathrm{LP}_{01}$ mode is initially relatively low, that is around $30 \%$, owing to the ratio gain to clad area. For this reason, the 7 and 19 cells TGF would provide higher confinement of the $\mathrm{LP}_{01}$ mode into the gain region for small pitch. With the increase of the pitch - and through it, of the gain area - the potential of the thermal gradient to confine strongly the light into the gain region grows, thus explaining why the singlemode range is shifted down in heat load. Due to its larger gain to clad area ratio, a 19 cells TGF offers an efficient singlemode operation for lower heat load but its range tends to be narrower by lack of HOM filtering. 


\subsection{Mode Field Area in thermally guiding fibers}

In order to quantify the strength of the thermal induced RIC, it appears interesting to look on the Mode Field Area (MFA) of the $\mathrm{LP}_{01}$ mode and to compare it with the extension of the gain area. Thus, one can stated that the fundamental mode guidance is mainly governed by the RIC induced into the gain region when the MFA gets smaller than the gain area $\mathrm{A}_{\text {gain }}$. Fig.4 displays the evolution of the fundamental mode MFA as a function of the pitch $\Lambda$ and the heat load ' $q$ '. The position of the limit MFA $=\mathrm{A}_{\text {gain }}$ is indicated by a thick white line. It appears that unique cell thermally guiding fibers experience a slower reduction of the MFA than a 7 or 19 cells structure. This is congruent with the fact that at constant pitch, this design exhibits a smaller extension of its gain area in comparison to the two others TGF.

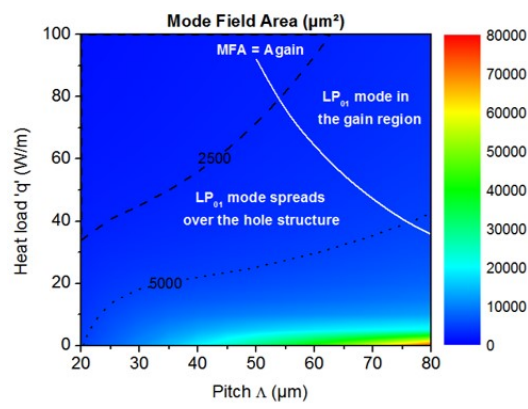

(a)

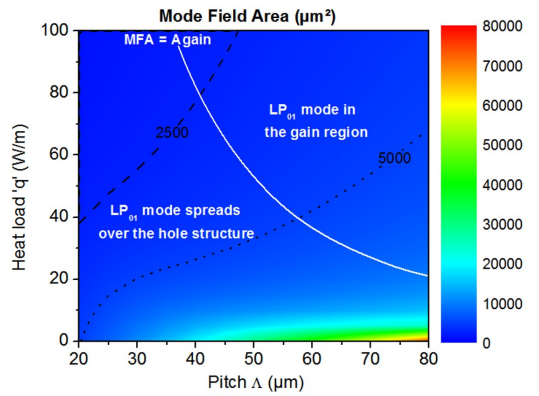

(b)

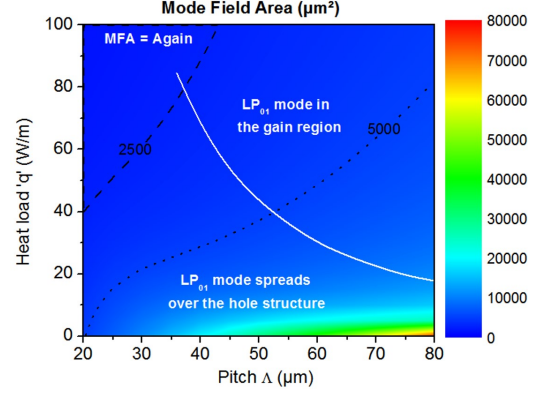

(c)

Figure 4. Evolution of the LP01 MFA with the heat load 'q' and the pitch $\Lambda$ into (a) a 1 cell TGF, (b) 7 cells TGF and (c) 19 cells TGF. The white line depicts the limit when the MFA of the fundamental mode equals to the area of gain $\mathrm{A}_{\text {gain }}$. Isolines also report MFA of $2,500 \mu \mathrm{m}^{2}$ (dashed line) and $5,000 \mu \mathrm{m}^{2}$ (dashed line).

It is important to notice the position of the MFA $=\mathrm{A}_{\text {gain }}$ line. By comparing the Fig.3 and 4, One can observe that this condition is centred into the range of efficient singlemode amplification. This underlines the fact that even if the thermal load is in cause into the fundamental mode confinement, it also restricts the operating range of singlemodedness through the rapid confinement of the first HOM. One way to extend of the singlemode range is to exploit the inherent spatial mode filtering of microstructured fibers.

\section{AIR SILICA LPFS}

Thus, the state-of-the-art air-silica LPF design was used to identify how the efficient singlemode amplification range evolves through the introduction of the periodic cladding microstructuration. Intuitively, one can expect to shift down the condition of effective singlemodedness $\left(\Gamma_{01}=80 \%\right)$ thanks to the ability of the air hole to improve the confinement of the fundamental mode while it will be interesting to observe how the modal discrimination will be influenced.

\subsection{Contribution of the cladding microstructuration}

The realization of an air-silica LPF is achieved by introducing a photonic crystal cladding onto the TGF design discussed beforehand. The LPF structures are depicted in Fig.5 together with the underlying stack lattice. Quantity are defined as previously and the air hole size is referred to as $\mathrm{d} / \Lambda$. In order to highlight the inherent contribution of this inner cladding microstructuration on the efficient singlemode amplification range, the normalized hole size is first kept low and in accordance with typical value reported for manufactured fibers, that is $\mathrm{d} / \Lambda=0.1$.

Fig. 6 reports on the evolution of the fundamental mode overlap with the gain region for the three LPF designs studied. The graphical representation is identical to that used previously. Through the introduction of air holes, the fundamental mode overlap has been improved for each geometry, with an increase of $20 \%$ for the cold fibers $(\mathrm{q}=0 \mathrm{~W} / \mathrm{m})$. Although it is hard to observe the influence on 1 cell fibers, the enhancement is clearer 


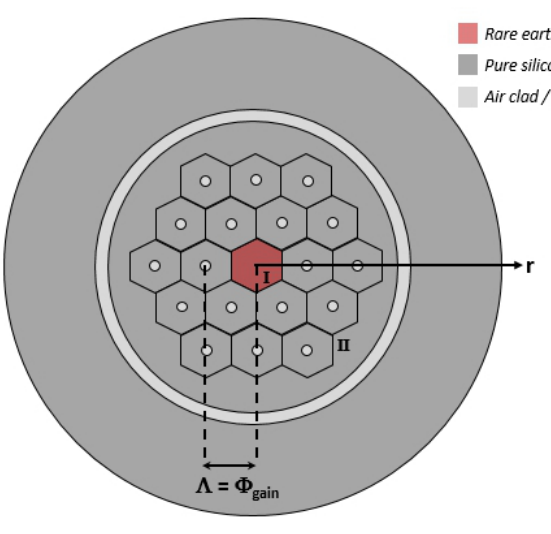

(a)

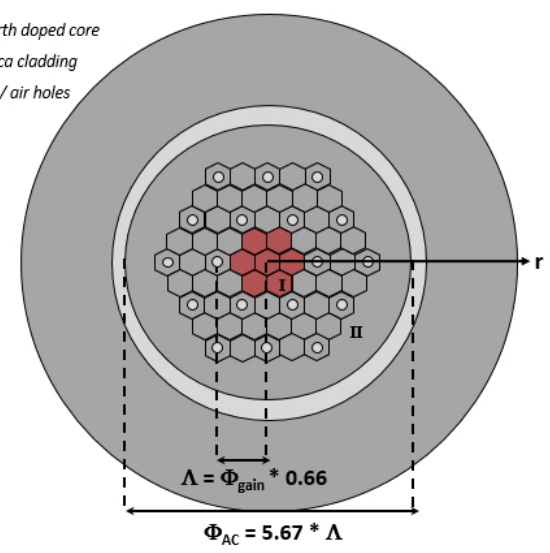

(b)

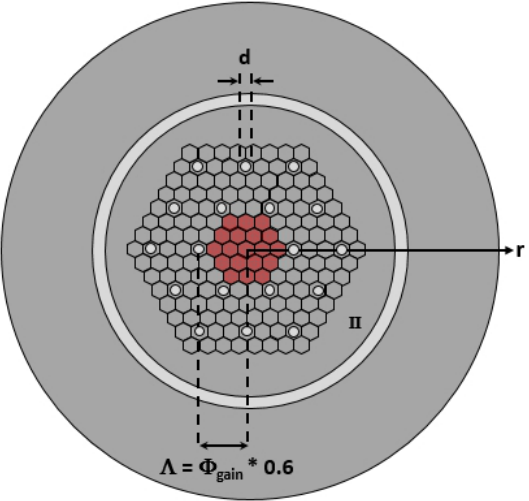

(c)

Figure 5. Graphical representations of Large-Pitch Fiber designs underlaid by the pattern of the initial stack. A periodic array of 18 air holes has been implemented over the design of Thermally Guiding Fibers discussed before. The red area represents the gain region, the pure silica is coloured in dark grey while the air holes/air-clad are in clear grey.

on the 7 and 19 cells designs. The singlemode ranges have been shifted down to lower heat load, meaning that both the $\mathrm{LP}_{01}$ and the most competitive $\mathrm{HOM}$ are better confined.

The most important fact is that the singlemode range has been widened thanks to the filtering ability of the cladding microstructuration. This effect is even more remarkable for the 19 cells LPF, whose the distance between the edge of the gain area and that of the closest air holes is minimal. In this configuration, the gain area spreading allows to benefit from the maximum of $\mathrm{LP}_{01}$ overlap since the singlemode limit has been shifted down as shown in Fig.6(c), while HOMs are still efficiently leaking in between the air holes. This emphasizes the prime importance the stack geometry can have. In fact, the gain area should spread as much as possible towards the air holes.

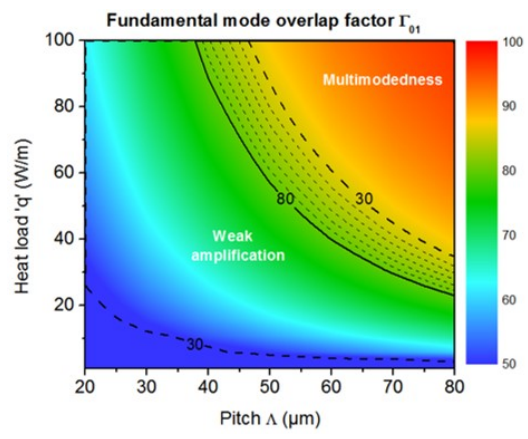

(a)

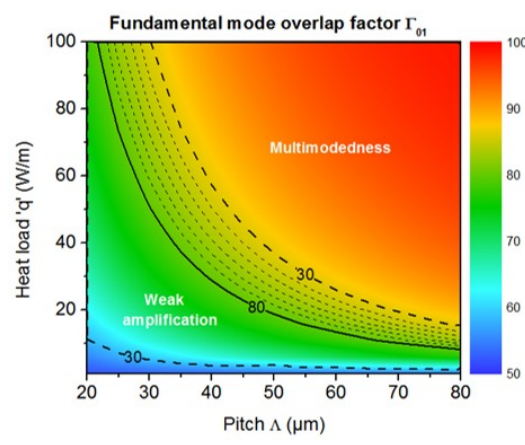

(b)

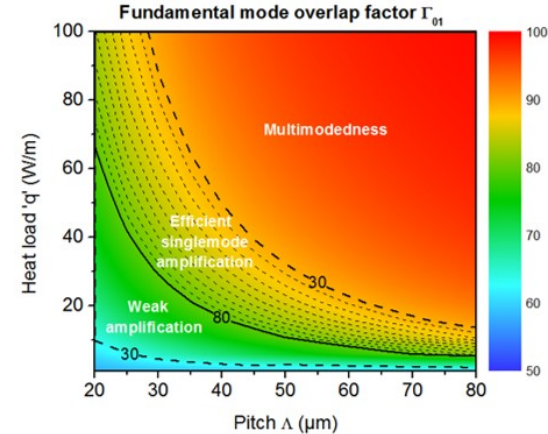

(c)

Figure 6. Evolution of the fundamental mode overlap factor with the heat load 'q' and the pitch $\Lambda$ into (a) a 1 cell LPF, (b) 7 cells LPF and (c) 19 cells LPF, the normalized air hole size of which are $\mathrm{d} / \Lambda=0.1$. The thick solid/dashed black lines represent respectively the limit of each abovementioned criteria: an efficient amplification $\Gamma_{01}=80 \%$ and an effective singlemodedness criteria: $\Delta \Gamma_{01}>80 \%$. The dashed area represents the region of efficient singlemode amplification.

\subsection{Influence of the normalized hole size}

Going further, it is interesting to tend toward standard normalized air hole sizes and observe the benefit on the fiber performances. Commonly, LPF employed for high-power operation exhibit a d/ $\Lambda$ of about $0.25-0.3$. Fig.7 
displays the evolution of the fundamental mode overlap factor into air-silica LPF with normalized air hole size of 0.3. The increase of the LP01 mode overlap factor through the air-holes enlargement is well denoted as the minimum overlap over the range of study is no longer falling under $80 \%$. Moreover, singlemode operation is now enabled into a 'cold' fiber, except for the 1 cell LPF which became clearly ineffective for the intended purpose. For what concerns the 7 and 19 active cells LPF, besides the reinforcement of the $\mathrm{LP}_{01}$, the range of effective singlemode amplification has been significantly broadened, although it allows for operation at lower heat load than with smaller air holes. This time, performances of these two LPF are quite equivalent. This points out the flexibility offered by air-silica LPF for tuning the range of singlemode operation.

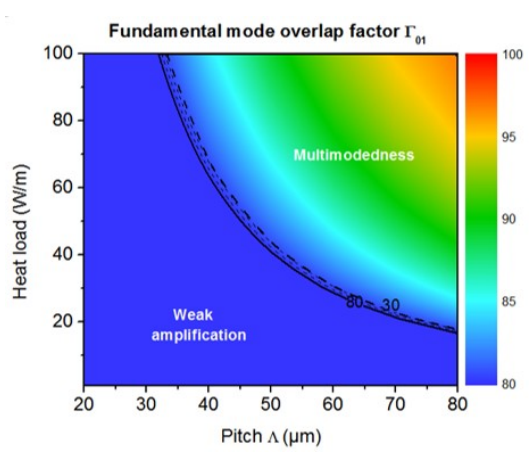

(a)

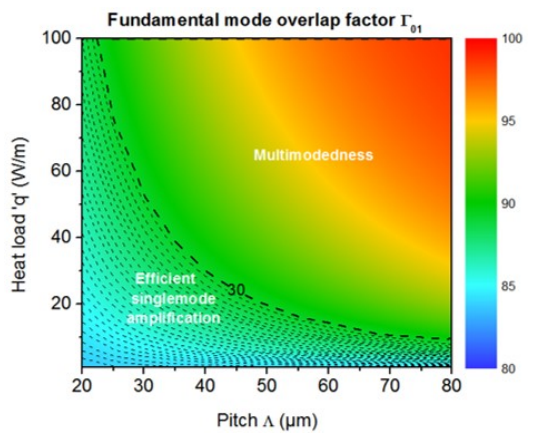

(b)

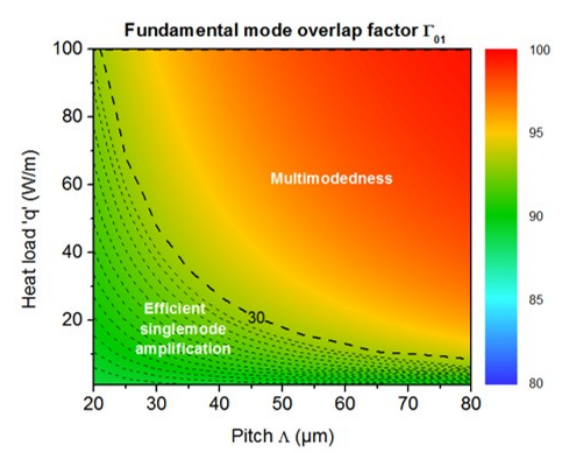

(c)

Figure 7. Evolution of the fundamental mode overlap factor with the heat load 'q' and the pitch $\Lambda$ into (a) a 1 cell LPF, (b) 7 cells LPF and (c) 19 cells LPF, the normalized air hole size of which are $\mathrm{d} / \Lambda=0.3$. The thick solid/dashed black lines represent respectively the limit of each abovementioned criteria: an efficient amplification $\Gamma_{01}=80 \%$ and an effective singlemodedness criteria: $\Delta \Gamma_{01}>80 \%$. The dashed area represents the region of efficient singlemode amplification.

Now, the singlemode range is no longer centred around the condition MFA $=\mathrm{A}_{\text {gain }}$ of the thermally guiding fiber. This means that although LPF structures exhibit a spatial mode filtering capacity, their performance at high power level are both limited by the thermal load and the ability of the cladding microstructuration in guiding HOM. Thus, next section will be treating structural evolutions of the inner cladding which has been shown as reinforcing the HOM rejection.

\section{POWER SCALING IN SYMMETRY-FREE AND APERIODIC VLMA FIBERS}

Noticeable improvements on the emitted beam quality have been recently demonstrated theoretically ${ }^{9,10}$ and experimentally ${ }^{12}$ on 19 cells fibers exhibiting a cladding symmetry reduction or suppression. However, the potential of these original structures for power scaling has only been discussed for symmetry-reduced cladding. Thus, it was intended to confront the performances of a symmetry-reduced PCF - Fig.8(a) - and an all-solid aperiodic LPF - Fig.8(b) - to that of the state-of-the-art air-silica LPF with a d/ $\Lambda=0.3$. The evolution of the fundamental mode into these two designs are shown in Fig.9(a-b). The upper limit of the singlemode operation into 19 cells air-silica LPF is reported on each graphs as a reference (white dashed line).

Interestingly, it is worth noting that each of these two non-periodic designs enables for a singlemode operation under higher heat load than air-silica LPF. Indeed, for a pitch of $50 \mu \mathrm{m}$, the upper limit of singlemodedness will reach $16.1 \mathrm{~W} / \mathrm{m}$ for an air-silica LPF, $25.4 \mathrm{~W} / \mathrm{m}$ with a symmetry-reduced PCF and $30 \mathrm{~W} / \mathrm{m}$ into a properly devised aperiodic LPF. This highlights the benefit of a cladding symmetry suppression for extending the singlemode range, and so, to push further the appearance of beam degradation. However, one may have noticed that the symmetry reduced PCF employed here does not satisfy the singlemode criteria for low heat load. This is because the FM suffers from mode couplings with cladding modes. For this reason, the inner cladding must be arranged in such way that cladding modes could not exhibit comparable effective index and significant spatial overlap with the FM. These objectives have driven the recent development of aperiodic LPF, such as the one depicted in Fig.8(b) or those reported in our previous work. ${ }^{10,12}$ Contrary to the symmetry reduced PCF, the aperiodic LPF has been devised so as to avert any mode coupling of the FM with cladding modes. Indeed, 


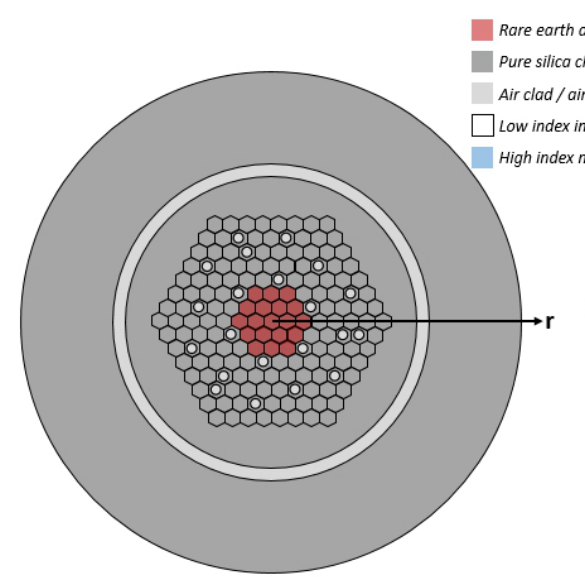

(a)

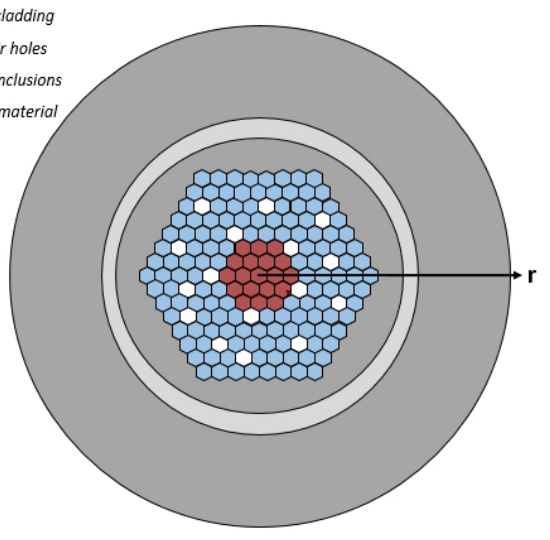

(b)

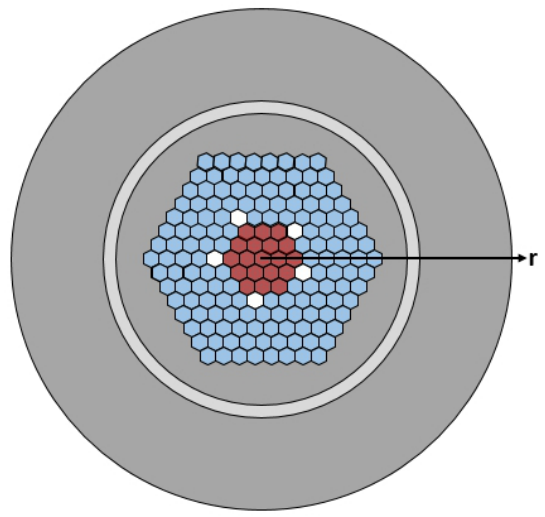

(c)

Figure 8. Graphical representations of (a) a Symmetry-free PCF (d/ $\Lambda=0.3$ ), (b) an all-solid aperiodic LPF made of solid inclusions $(\mathrm{d} / \Lambda=0.33)$ and $(\mathrm{c})$ an all-solid cladding reduced aperiodic $\operatorname{LPF}(\mathrm{d} / \Lambda=0.33)$. The initial stack geometry is underlaid on structures. The red area represents the gain region, the clear blue is a high index material index-matching the gain medium, the pure silica is coloured in dark grey while the air holes/air-clad are in white, solid low index.

the extension on the inner cladding, made of a high index material index matching the gain medium, is no longer limited by the air clad but by a silica layer. This aims in avoiding the guiding of annular cladding mode, the effective index of which can be close enough to the FM that i mode coupling may occur.

On Fig.9(b), one can observe that the criteria of effective amplification is satisfied over the whole range of investigation while the singlemode range has been broadened. As discussed before, such a design allows in increasing by half the range of effective singlemode operation in comparison to state-of-the-art air-silica LPF with $\mathrm{d} / \Lambda=0.3$ and to cover the whole range reachable by tuning the air holes size. So as to provide an insight on how this aperiodic cladding works, a Cladding-Reduced aperiodic LPF (CR-LPF) was devised and studied. On Fig.8(c), one can see that only the 5 low inclusions in the closest vicinity of the gain region have been preserved with the intention to get understanding on the modal filtering ability of this first layer. The fundamental mode

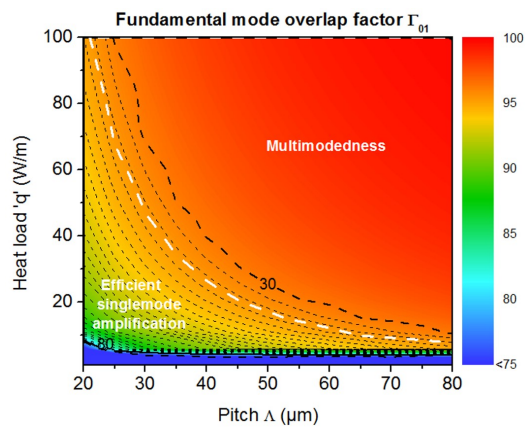

(a)

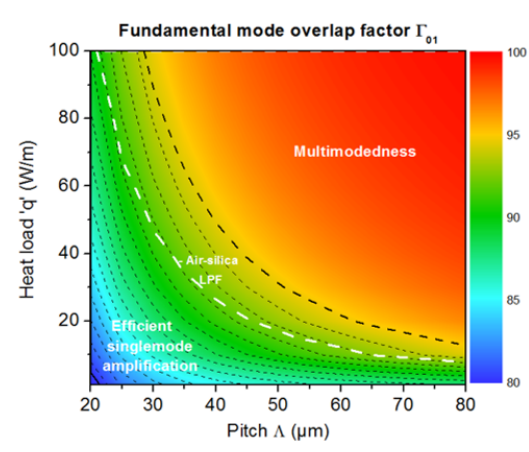

(b)

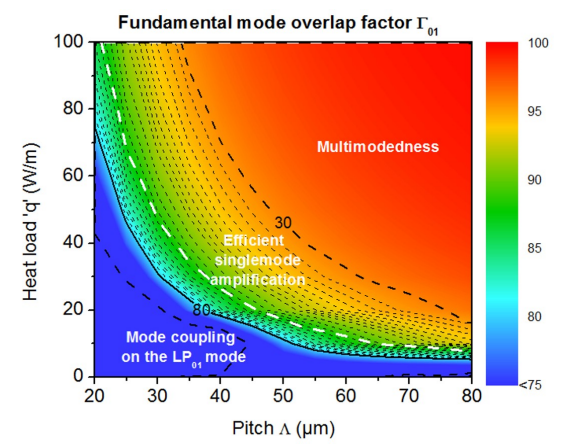

(c)

Figure 9. Evolution of the fundamental mode overlap factor with the heat load 'q' and the pitch $\Lambda$ into a) a Symmetryreduced PCF - d $/ \Lambda=0.25-$, (b) an all-solid aperiodic LPF - d $/ \Lambda=0.33$ - and (c) an equivalent all-solid cladding reduced aperiodic LPF - d $/ \Lambda=0.33$-. The thick solid/dashed black lines represent respectively the limit of each abovementioned criteria: an efficient amplification $\Gamma_{01}=80 \%$ and an effective singlemodedness criteria: $\Delta \Gamma_{01}>80 \%$. The dashed area represents the region of efficient singlemode amplification and the thick dashed white line reports the upper limit of the singlemode range into a 19 cells air-silica $\mathrm{LPF}$ with $\mathrm{d} / \Lambda$ of 0.33 . 
overlap factor of this CR-LPF have been computed over the whole range of analysis. First of all, because of the small number of inclusions paving the inner cladding, it is not surprising to observe modes couplings on the FM for low heat load. Nevertheless, the upper limit of the singlemode range appears to be higher than into the aperiodic LPF. This asserts that these 5 inclusions allows for an efficient FM overlap factor with the gain region as well as an effective filtering of the HOM. Hence, the inner cladding must be completed by introduced inclusions in such way that they are impacting as little as the modes filtering behaviour of the first layer while the reduced the effective index of cladding modes, dodging the possibilities of modes coupling on the FM. The all-aperiodic LPF design reported in this section is one of those enabling to offer an efficient singlemode amplification at the highest heat load without risking mode coupling.

\section{CONCLUSION}

We reported in this article a comparative analysis of the heat load influence into VLMA fibers. First, the performance of 'Thermally Guiding Fibers' have been investigated, enabling to demonstrate that even without any cladding microstructuration, an efficient singlemode amplification can be performed into these TGF. Then, the contribution of a cladding microstructuration for high-order-modes filtering has been evidenced, offering a noteworthy widening of the singlemode range for normalized air hole of about 0.3 . The highest thermal load sustainable by this structure can be adjusted through the air holes size. Thus, air-silica LPF can be slight tailored so as to fulfil user request in term of power range. The stack geometry has also been discussed, evidencing the benefit of 19 cells design. At last, a significant broadening of the efficient singlemode amplification range was shown by using an aperiodic cladding structure and by limiting the extent of the inner cladding. Hence, additional inclusions are been placed in such way to impact the cladding modes, avoiding modes coupling on the FM, and reduce as little as possible the mode filtering ability of the whole design. Further work in allowing efficient singlemode amplification under higher heat load are in progress.

\section{ACKNOWLEDGMENTS}

This work, conducted under the AVANTAGE project, was co-funded by the European Union and Eolite Systems. EC is involved in the Région Limousin with the "Fonds européen de développement économique et régional".

\section{REFERENCES}

[1] Stiles, E., "Recent Progress in Fiber Lasers," in 5th international fiber laser workshop, 1-11 (2009).

[2] Hädrich, S., Klenke, A., Hoffmann, A., Eidam, T., Gottschall, T., Rothhardt, J., Limpert, J., and Tünnermann, A., "Nonlinear compression to sub-30-fs $0.5 \mathrm{~mJ}$ pulses at $135 \mathrm{~W}$ of average power," Optics Letter 38(19), 3866-3869 (2013).

[3] Mattsson, K. E., "Photo darkening of rare earth doped silica," Optics express 19, 19797-812 (2011).

[4] Koponen, J., Laurila, M., and Hotoleanu, M., "Inversion behavior in core- and cladding-pumped Yb-doped fiber photodarkening measurements," Applied optics 47(25), 4522-4528 (2008).

[5] Jauregui, C., Eidam, T., Otto, H.-J., Stutzki, F., Jansen, F., Limpert, J., and Tünnermann, A., "Physical origin of mode instabilities in high-power fiber laser systems," Optics express 20, 12912-25 (2012).

[6] Engholm, M., Jelger, P., Laurell, F., and Norin, L., "Improved photodarkening resistivity in ytterbium-doped fiber lasers by cerium codoping," Optics letters 34(8), 1285-1287 (2009).

[7] Dong, L., Mckay, H. A., Marcinkevicius, A., Fu, L., Li, J., Thomas, B. K., and Fermann, M. E., "Extending effective are of fundamental mode in optical fibers," Journal of Lightwave Technology 27(11), 1565-1569 (2009).

[8] Limpert, J., Stutzki, F., Jansen, F., Otto, H.-J., Eidam, T., Jauregui, C., and Tünnermann, A., "Yb-doped large-pitch fibres: effective single-mode operation based on higher-order mode delocalisation," Light: Science E) Applications 1, e8 (2012).

[9] Coscelli, E., Molardi, C., Cucinotta, A., and Selleri, S., "Symmetry-free Tm-doped photonic crystal fiber with enhanced mode area," IEEE Journal of Selected Topics in Quantum Electronics , 1077-260X (2014). 
[10] Dauliat, R., Jamier, R., Schuster, K., Grimm, S., and Roy, P., "Striving towards a periodicity breaking of the photonic crystal cladding microstructuration for an unprecedented singlemode robustness," in Proc. SPIE 9128, Micro-structured and Specialty Optical Fibres III, (0), 912807 (2014).

[11] Stutzki, F., Jansen, F., Jauregui, C., Limpert, J., and Tünnermann, A., "Non-hexagonal Large-Pitch Fibers for enhanced mode discrimination," Optics express 19, 12081-6 (2011).

[12] Benoît, A., Dauliat, R., Jamier, R., Humbert, G., Grimm, S., Schuster, K., Salin, F., and Roy, P., "Highly efficient higher-order modes filtering into aperiodic very large mode area fibers for single-mode propagation," Optics letters 39, 4561-4 (2014).

[13] Jansen, F., Stutzki, F., Otto, H.-J., Baumgartl, M., Jauregui, C., Limpert, J., and Tünnermann, A., "The influence of index-depressions in core-pumped Yb-doped large pitch fibers," Optics express 18, 26834-42 (2010).

[14] Jansen, F., Stutzki, F., Otto, H.-j., Jauregui, C., Limpert, J., and Tünnermann, A., "High-power thermally guiding index-antiguiding-core fibers," Optics letters 38(4), 510-512 (2013).

[15] Benoît, A., Dauliat, R., Schuster, K., Grimm, S., Jamier, R., and Roy, P., "Optical fiber microstructuration for strengthening single-mode laser operation in high power regime," Optical Engineering 53(7), 071817 (2014).

[16] Dauliat, R., Gaponov, D., Benoît, A., Salin, F., Schuster, K., Jamier, R., and Roy, P., "Inner cladding microstructuration based on symmetry reduction for improvement of singlemode robustness in VLMA fiber," Optics Express 21(16), 18927-18936 (2013).

[17] Jø rgensen, M. M., Petersen, S. R., Laurila, M., Læ gsgaard, J., and Alkeskjold, T. T., "Optimizing single mode robustness of the distributed modal filtering rod fiber amplifier," Optics express 20(7), 7263-7273 (2012). 